\title{
Charge-transfer interaction of 4,13-diamino[2.2]paracyclophane with $\pi$-acceptors
}

\author{
Kamal M. El-Shaieb ${ }^{a}$, Aboul-Fetouh E. Mourad ${ }^{* a}$, Ashraf A. Aly ${ }^{a}$, and Henning Hopf ${ }^{b}$ \\ ${ }^{a}$ Chemistry Department, Faculty of Science, El-Minia University, El-Minia, Egypt \\ ${ }^{b}$ Institut für Organische Chemie, Technische Universität Braunschweig, Hagenring 30, D-38106 \\ Braunschweig, Germany \\ E-mail: mouradaboulf@yahoo.com
}

\begin{abstract}
4,13-Diamino[2.2]paracyclophane (1) reacts with different $\pi$-acceptors via charge transfer complexation providing substituted $N$-amino derivatives. Depending on the donor/acceptor combination, mono and/or di-substituted $N$-derivatives have been obtained.
\end{abstract}

Keywords: 4,13-Diamino[2.2]paracyclophane, charge transfer complexation, $\pi$-acceptors

\section{Introduction}

[2.2]Paracylophanes are serving as excellent donating systems for charge-transfer (CT) complexation comparable to classical aromatic compounds, and it has been proven that this behavior is mainly due to the presence of transannular electronic interactions between the two benzene rings in the cyclophane molecule. ${ }^{1-3}$ In recent years we reported on the synthesis of some interesting new heterocyclic systems by CT-complexation between simple heterocycles as electron donors and various $\pi$-acceptors. ${ }^{4-13}$ In 1993 Mourad et al ${ }^{14}$ described the anomalous behavior of 4-amino[2.2]paracyclophane and its $N$-methyl derivative towards tetracyanoethylene (TCNE) and 2,3-dichloro-5,6-dicyano-1,4-benzoquinone (DDQ), giving unexpected products such as 2-(4-[2.2]-paracyclophanyl)-3,3-dicyanoxaziridine, 4-( $N$-carbonitril- $N$ ethyl)amino[2.2]paracyclophane as well as 2,3-dichloro-5-cyano-6([2.2]paracyclophanyl)amino-1,4-benzoquinone.

Recently, we have furthermore succeeded to construct a variety of poorly investigated types of heterocyclic compounds such as pleiadenes and perimidines, by the reaction of 1,8 diaminonaphthalene with selected $\pi$-acceptors. ${ }^{15}$

In the light of these promising results we turned our attention to 4,13-diamino[2.2]paracyclophane (1) ${ }^{16}$ as another electron donor to investigate its donating properties towards electron acceptors such as 7,7,8,8-tetracyanoquinodimethane (TCNQ, 2), 2-dicyano- 
methyleneindan-1,3-dione (CNIND, 3), 2,3-dichloro-1,4-naphthoquinone (DCHNQ, 4), 2,3dicyano-1,4-naphthoquinone (DCNQ, 5), and 2,3-dichloro-5,6-dicyano-1,4-benzoquinone $(\mathrm{DDQ}, \mathbf{6})$. The results of the reactions between $\mathbf{1}$ and these acceptors are described herein.

\section{Results and Discussion}

Addition of the electron donor 1 to either of the electron acceptors 2-6 in dichloromethane leads to complex formation as characterized by a CT-band appearing in the visible region (Table 1, Experimental). The CT-absorption gradually disappears to give rise to the formation of new reaction products.

Interaction of TCNQ 2 with 1 furnished a single product to which structure $7,7-\left(4^{\prime}, 13^{\prime}\right.$ diamino[2.2]paracyclophanyl)-8,8-dicyanoquinodimethane (7) was assigned on the basis of the spectroscopic data as well as elemental analysis (Scheme 1). Both MS and elemental analysis confirm the molecular formula of 7 as $\mathrm{C}_{26} \mathrm{H}_{20} \mathrm{~N}_{4}$. The symmetry of compound 7 results in simplification of its NMR spectra. The ${ }^{1} \mathrm{H}$ NMR spectrum reveals besides the signals due to the aromatic and ethylenic protons in their expected positions a broad signal at $\delta=10.92$ due to the two $\mathrm{NH}$ groups as well as the absence of the signals of the $\mathrm{NH}_{2}$ groups. Also the ${ }^{13} \mathrm{C} \mathrm{NMR}$ spectrum showed the existence of fifteen carbon atoms including only three signals; two for the $\mathrm{CH}_{2}$ carbon and the other for the cyano group. On the basis of structural features of both TCNQ and CNIND, the reaction of 1 with CNIND (3) should follow the same sequence as that with 2. However, only one amino group of $\mathbf{1}$ participated in the reaction, in which one equivalent of HCN was eliminated to give the adduct $\mathbf{8}$ (Scheme 1).

This could be rationalized in terms of the stability of the mono-substituted product $\mathbf{8}$, due to the intramolecular hydrogen bond which is further substantiated from the down field shift of the $\mathrm{NH}$ group $(\delta=11.20)$, as well as, the $\widetilde{\mathrm{v}}_{\max }$ of this group at $3438-3355 \mathrm{~cm}^{-1}$. Another interesting future of $\mathbf{1}$ was observed when it was subjected to react with 1,4-naphtho- and benzoquinones 4, 5, and $\mathbf{6}$ to give mono- and di-substituted products. Of particular interest is the formation of the reaction products 10,12 and 14, in which the two hydrogen atoms of the same amino group are replaced by two electron acceptor molecules. Surprisingly, the highly sterically hindered reaction products $\mathbf{1 0}, \mathbf{1 2}$ and 14 are formed rather than the products expected by substitution of only one hydrogen atom in both amino groups in $\mathbf{1}$ by an electron acceptor molecule. 


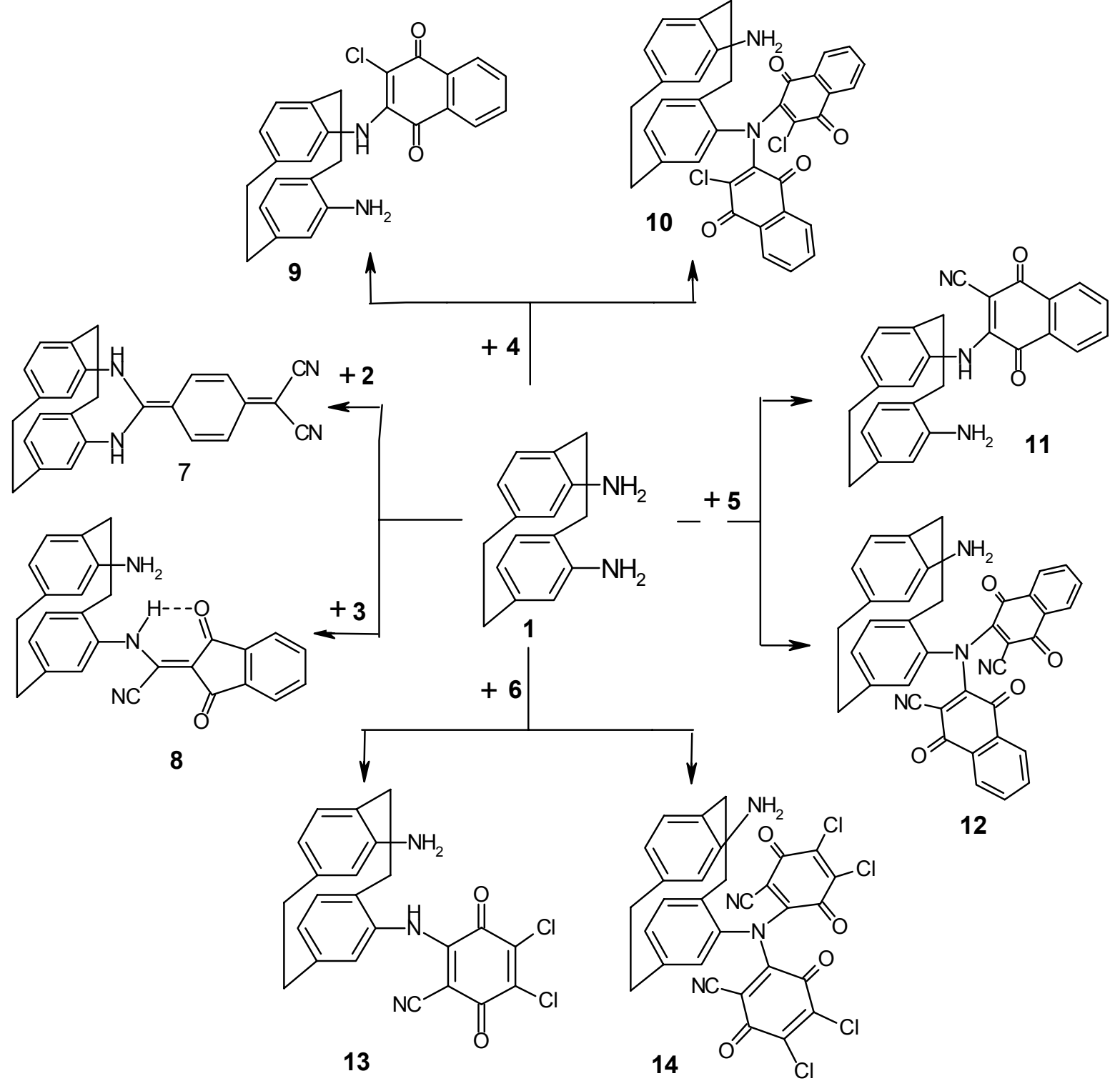

\section{Scheme 1}

The attack of the two molecules of the acceptor at the same nitrogen atom to give compounds 10, 12 and 14 rather than forming compound 15 may be rationalized in terms of the stability of the resonance structures 17-19 (Figure 1). It is evident that in structure 16, the two positively charged nitrogen atoms are located in a pseudo-geminal position so they are so close to each to make this alternative adduct instable because of electronic repulsion. On the other hand, in structures 18 and 19, the lone pair of electrons on the di-substituted nitrogen atom enters into conjugation with the two-quinone moieties. 

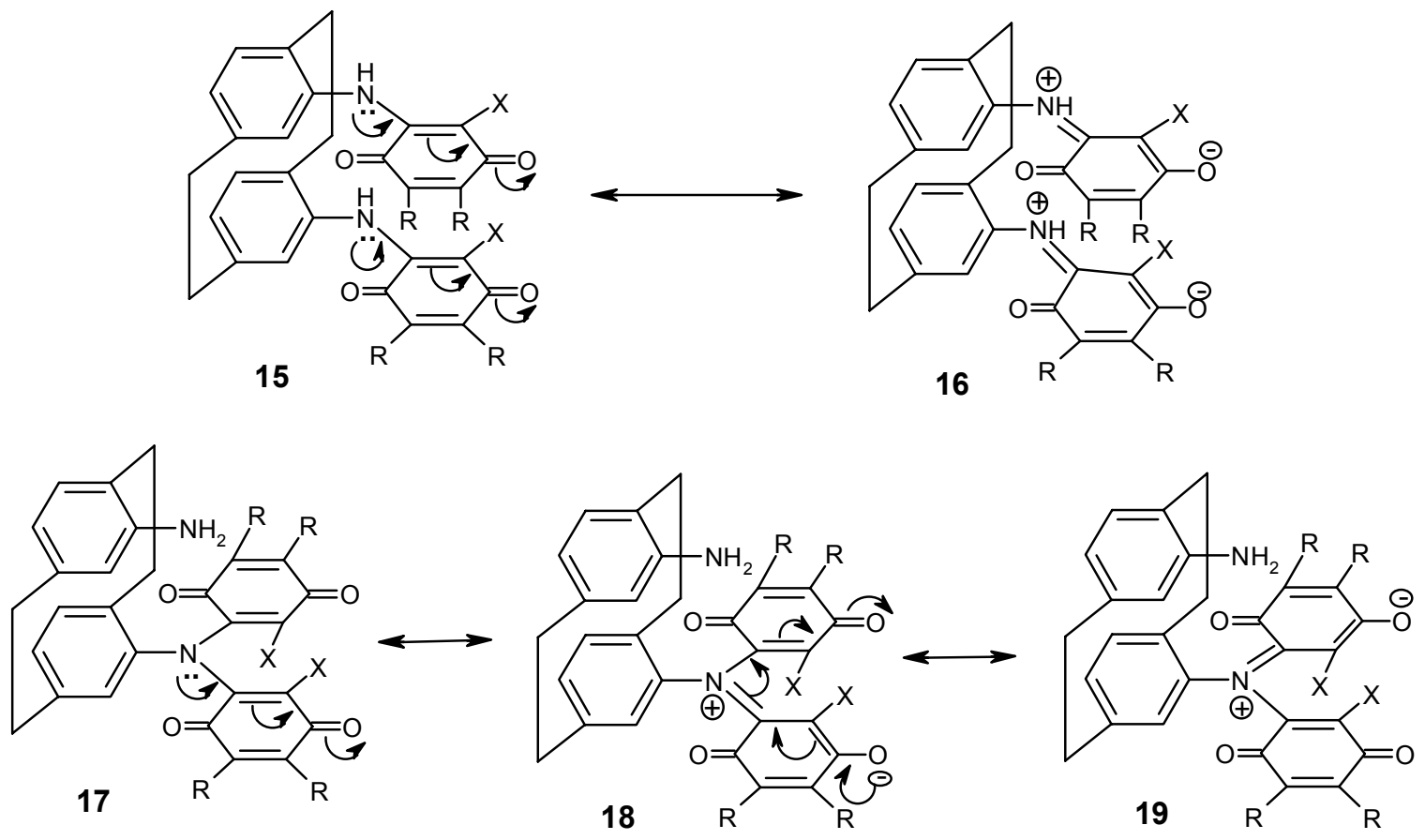

Figure 1

Consequently this nitrogen atom becomes highly positively charged and the resulting structure is stabilized due to the location of the free amino group with its lone pair just opposite of the positive charge generating a new donor-acceptor interaction.

The structures of the mono-substituted products $\mathbf{9 , 1 1}$ and $\mathbf{1 3}$ are fully supported by this analytical and spectroscopic data. Besides the signals due to the aromatic protons in the paracyclophane and naphthoquinone moieties, their ${ }^{1} \mathrm{H}$ NMR spectra are characterized by the presence of two broad bands in the range of $\delta=9.90-11.00$ and 3.50-3.70, assigned to the $\mathrm{NH}$ and $\mathrm{NH}_{2}$ groups respectively. The ${ }^{13} \mathrm{C} \mathrm{NMR}$ spectra are also in agreement with the proposed structures. Furthermore, mass spectra and elemental analyses give strong evidence for the molecular formula of the reaction products. Of great significance in the structural proof of the disubstituted products 10,12 and 14 are their ${ }^{1} \mathrm{H}$ NMR spectral data, that revealed, besides signals due to the aromatic and methylene protons, a broad singlet in the range of $\delta=3.70-3.93$ assignable to the $\mathrm{NH}_{2}$ groups. Besides the ${ }^{1} \mathrm{H}$ NMR spectra of compounds 10, 12 and 14, these structures were further substantiated by means of ${ }^{13} \mathrm{C} N M R$, mass spectra as well as elemental analysis.

In conclusion, the results described in this paper reveal for the first time that structurally novel adducts are formed when one of the components in these CT-complexes has a [2.2]paracyclophane structure. 


\section{Experimental Section}

General Procedures. Melting points are uncorrected. NMR spectroscopy: Bruker AM-400, solvent $\mathrm{CDCl}_{3}$, internal standards: TMS $(\delta=0.00)$ for ${ }^{1} \mathrm{H}, \mathrm{CDCl}_{3}(\delta=77.05)$ for ${ }^{13} \mathrm{C} \mathrm{NMR}$. Column chromatography: silica gel 7714 (Merck). For preparative layer chromatography (PLC), glass plates $(20 \times 48 \mathrm{~cm})$ were covered with a slurry of silica gel Merck $\mathrm{PF}_{254}$ air-dried using the solvents listed for development. Zones are detected by quenching of indicator fluorescence upon exposure to $254 \mathrm{~nm}$ UV light. Elemental analyses were performed by Institute für Anorganische Chemie, Technische Universität Braunschweig. Mass spectra were recorded on a Finnigan MAT 8430 spectrometer at $70 \mathrm{eV}$. IR spectra were obtained on Nicolet 320 FT-IR instruments using $\mathrm{KBr}$ pellets and paraffin films.

Materials. 7,7,8,8-Tetracyanoquinodimethane (TCNQ, 2), and 2,3-Dichloro-1,4-naphthoquinone (DCHNQ, 4) were bought from Fluka. 2-Dicyanomethyleneindane-1,3-dione (CNIND, 3) was prepared according to the procedure described by Chatterjee. ${ }^{17}$ 2,3-Dicyano-1,4-naphthoquinone (DCNQ, 5) was prepared from 4 according to ref. 18. 2,3-Dichloro-5,6-dicyano-1,4benzoquinone (DDQ, 6), was a commercial product from (Aldrich). 4,13Diamino[2.2]paracyclophane (1) was prepared according to ref. 16.

General synthetic procedure. To a solution of $1 \mathrm{mmol}$ of the acceptor (2-8) in $25 \mathrm{~mL}$ of dry dichloromethane, a solution of $\mathbf{1}(1 \mathrm{mmol})$ in $25 \mathrm{~mL}$ of dichloromethane was added with stirring. The solvent was concentrated and the residue was applied to PLC using toluene as the eluent. Whereas compounds $\mathbf{7}$ and $\mathbf{8}$, appeared as the only separated zones, for adducts 9-14, two zones were obtained, the mono-substituted derivative was moving faster than the di-substituted one. The following Table summarizes the reaction conditions for the reaction of $\mathbf{1}$ with 2-6 and the CT-absorption maxima for their CT-complexes.

Table 1. Reaction conditions for the reaction of $\mathbf{1}$ with $\pi$-acceptors $\mathbf{2 - 6}$, and the CT-absorption maxima for their CT-complexes in dichloromethane at $22{ }^{\circ} \mathrm{C}$

\begin{tabular}{|l|l|l|l|}
\hline Acceptor & Reaction time $(\mathrm{h})$ & Temperature $\left({ }^{\circ} \mathrm{C}\right)$ & $\lambda_{\max }$ of the CT-complexes $(\mathrm{nm})$ \\
\hline TCNQ (2) & 12 & 22 & 608 \\
\hline CNIND (3) & 24 & 22 & 468 \\
\hline DCHNQ (4) & 40 & 22 & 500 \\
\hline DCNQ (5) & 24 & 22 & 755 \\
\hline DDQ (6) & 14 & 22 & 711 \\
\hline
\end{tabular}

7,7(4',13'-Diamino[2.2]paracyclophanyl)-8,8-dicyanoquinodimethane (7). Red crystals (acetonitrile) $\left(\mathrm{mp}>300{ }^{\circ} \mathrm{C}, 39 \mathrm{mg}, 65 \%\right)$; IR(KBr): $\widetilde{\mathrm{v}}_{\max }=3438 \mathrm{~cm}^{-1}(\mathrm{NH}), 3070-3010$ (Ar$\mathrm{CH}), 2989-2860$ (aliph.-CH), $2220(\mathrm{CN}) ;{ }^{1} \mathrm{H}$ NMR (DMSO-d 6 ); $\delta 3.35-3.42\left(8 \mathrm{H}, \mathrm{m}, 2 \mathrm{CH}_{2}-\mathrm{CH}_{2}\right)$, $6.55(2 \mathrm{H}, \mathrm{d}, J=1.38 \mathrm{~Hz}, \mathrm{Ar}-\mathrm{H}), 6.59(2 \mathrm{H}, \mathrm{d}, J=1.38 \mathrm{~Hz}, \mathrm{Ar}-\mathrm{H}), 6.73(2 \mathrm{H}, \mathrm{s}, \mathrm{Ar}-\mathrm{H}), 6.95$ (2H, $\mathrm{d}, J=8.66 \mathrm{~Hz}, \mathrm{TCNQ}), 7.70(2 \mathrm{H}, \mathrm{d}, J=8.66 \mathrm{~Hz}, \mathrm{TCNQ}), 10.92(2 \mathrm{H}, \mathrm{br}, \mathrm{s}, 2 \mathrm{NH}) ;{ }^{13} \mathrm{C}$ NMR 
$\left(\mathrm{DMSO}_{\mathrm{d}}\right) ; \delta 30.50\left(\mathrm{CH}_{2}\right), 34.72\left(\mathrm{CH}_{2}\right), 115.04(\mathrm{CN}), 117.55,118.25,123.35,128.83,129.82$, 132.90, 134.43, 135.15, 136.43, 142.04, 149.84, $159.54($ Ar-C); MS (70 eV): m/z (\%) = 388 $\left[\mathrm{M}^{+}\right]$(100), 363 (10), 270 (22), 248 (10), 205 (32), 204 (20), 130 (12), 119 (10), 103 (4), 77 (10), 57 (6); Anal. Calcd for $\mathrm{C}_{26} \mathrm{H}_{20} \mathrm{~N}_{4}$ : C, 80.39; H, 5.19; N, 14.42. Found: C, 80.25; H, 5.30; N, $14.30 \%$.

4-Amino-13(2'-cyanomethyleneindane-1',3'-dionyl)amino[2.2]paracyclophane $\quad(8) . \quad$ Red needles (ethanol) (mp $241{ }^{\circ} \mathrm{C}, 38 \mathrm{mg}, 67 \%$ ); IR (KBr): $\widetilde{\mathrm{v}}_{\max }=3438-3355 \mathrm{~cm}^{-1}\left(\mathrm{NH}, \mathrm{NH}_{2}\right)$, 3067-3012 (Ar-CH), 2969-2852 (aliph.-CH), 2220 (CN), 1701 (CO); ${ }^{1} \mathrm{H}$ NMR ( $\left.\mathrm{CDCl}_{3}\right) ; \delta 2.74-$ $3.30\left(8 \mathrm{H}, \mathrm{m}, 2 \mathrm{CH}_{2}-\mathrm{CH}_{2}\right), 3.42\left(2 \mathrm{H}, \mathrm{s}, \mathrm{NH}_{2}\right), 5.72(1 \mathrm{H}, \mathrm{s}, \mathrm{Ar}-\mathrm{H}), 6.38(1 \mathrm{H}, \mathrm{d}, J=7.60 \mathrm{~Hz}, \mathrm{Ar}-$ H), $6.44(1 \mathrm{H}, \mathrm{d}, J=7.60 \mathrm{~Hz}, \mathrm{Ar}-\mathrm{H}), 6.47(1 \mathrm{H}, \mathrm{d}, J=7.70 \mathrm{~Hz}, \mathrm{Ar}-\mathrm{H}), 6.61$ (1H, s, Ar-H), 7.607.88 (4H, m, CNIND), $11.20(1 \mathrm{H}, \mathrm{br}, \mathrm{s}, \mathrm{NH}) ;{ }^{13} \mathrm{C} \mathrm{NMR}\left(\mathrm{CDCl}_{3}\right) ; \delta 28.60,32.11,34.70,34.80$ $\left(2 \mathrm{CH}_{2}-\mathrm{CH}_{2}\right), 108.06,109.50,111.60,112.18,114.40,117,124.00,127.40,128.00,128.04$, $129.00,129.70,130.16,130.50,131.16,134.80,135.90,136.80,139.04$ (Ar-C), 141.04 (NC-C$\mathrm{NH}), 145.89$ (CO-C-CO), 186.31 (CO), 189.15 (CO); MS (70 eV): m/z (\%)= $419\left[\mathrm{M}^{+}\right](20), 392$ (100), 273 (50), 247 (20), 217 (14), 189 (12), 165 (10), 145 (8), 119 (50), 91 (10), 77 (12); Anal. Calcd for $\mathrm{C}_{27} \mathrm{H}_{21} \mathrm{~N}_{3} \mathrm{O}_{2}$ : C, 77.31; H, 5.05; N, 10.02. Found: C, 77.10; H, 5.03; N, 9.99\%.

2-N-[4'(13'-Amino[2.2]paracyclophanyl)]amino-3-chloro-1,4-naphthoquinone (9). Red crystals (ethanol) $\left(\mathrm{mp} 170{ }^{\circ} \mathrm{C}, 20 \mathrm{mg}, 36 \%\right.$ ); IR (KBr): $\widetilde{\mathrm{v}}_{\max }=3428,3366 \mathrm{~cm}^{-1}\left(\mathrm{NH}, \mathrm{NH}_{2}\right)$, 3065-3011 (Ar-CH), 2986-2865 (aliph.CH), 1719, 1675 (2CO); ${ }^{1} \mathrm{H}$ NMR $\left(\mathrm{CDCl}_{3}\right) ; \delta$ 2.70-3.50 $\left(8 \mathrm{H}, \mathrm{m}, 2 \mathrm{CH}_{2}-\mathrm{CH}_{2}\right), 3.70\left(2 \mathrm{H}, \mathrm{br}, \mathrm{s}, \mathrm{NH}_{2}\right), 5.20(1 \mathrm{H}, \mathrm{s}, \mathrm{Ar}-\mathrm{H}), 5.78(1 \mathrm{H}, \mathrm{d}, J=7.60 \mathrm{~Hz}, \mathrm{Ar}-\mathrm{H})$, $6.06(1 \mathrm{H}, \mathrm{dd}, J=7.60,1.50 \mathrm{~Hz}, \mathrm{Ar}-\mathrm{H}), 6.21(1 \mathrm{H}, \mathrm{d}, J=7.60, \mathrm{Ar}-\mathrm{H}), 6.39(1 \mathrm{H}, \mathrm{d}, J=1.52 \mathrm{~Hz}$, Ar-H), $6.54(1 \mathrm{H}, \mathrm{d}, J=7.60 \mathrm{~Hz}, \mathrm{Ar}-\mathrm{H}), 6.96-7.72(2 \mathrm{H}, \mathrm{m}, \mathrm{DCHNQ}), 7.98-8.08$ (2H, m, DCHNQ), $9.90(1 \mathrm{H}, \mathrm{br}, \mathrm{s}, \mathrm{NH}) ;{ }^{13} \mathrm{C} \mathrm{NMR}\left(\mathrm{CDCl}_{3}\right) ; \delta 29.63,32.13,34.66,35.12\left(2 \mathrm{CH}_{2}-\mathrm{CH}_{2}\right)$, $122.40,122.81,125.33,125.73,126.74,127.00,128.82,132.76,132.95,133.24,133.70,133.90$, 134.32, 134.40, 134.61, 135.61, 135.95, 140.22, 140.91, 148.52 (Ar-C), 179.40 (CO), 180.06 $(\mathrm{CO}) ; \mathrm{MS}(70 \mathrm{eV}): \mathrm{m} / \mathrm{z}(\%)=430\left[\mathrm{M}^{+2}\right](40), 428\left[\mathrm{M}^{+}\right](90), 392$ (12), 308 (16), 273 (100), 217 (20), 149 (26), 144 (30), 119 (40), 91 (12), 77 (10); Anal. Calcd for $\mathrm{C}_{26} \mathrm{H}_{21} \mathrm{ClN}_{2} \mathrm{O}_{2}$ : C, 72.81; $\mathrm{H}$, 4.93; N, 6.53. Found: C, 72.70; H, 4.85; N, 6.45\%.

4-Amino-13- $\mathrm{N}, \mathrm{N}$-bis-2'-(3'-chloro-1,4-naphthoquinonyl)amino[2.2]paracyclophane $\quad(10)$. Violet crystals (toluene) (mp $260{ }^{\circ} \mathrm{C}, 7 \mathrm{mg}, 19 \%$ ); IR (KBr): $\widetilde{v}_{\max }=3433 \mathrm{~cm}^{-1}\left(\mathrm{NH}_{2}\right), 3060-$ 3014 (Ar-CH), 2985-2860 (aliph.- $\mathrm{CH}), 1718,1671$ (2CO); ${ }^{1} \mathrm{H} \mathrm{NMR}\left(\mathrm{CDCl}_{3}\right) ; \delta$ 2.60-3.52 (8H, m, $\left.2 \mathrm{CH}_{2}-\mathrm{CH}_{2}\right), 3.93\left(2 \mathrm{H}\right.$, br. s, $\left.\mathrm{NH}_{2}\right), 5.22(1 \mathrm{H}, \mathrm{s}, \mathrm{Ar}-\mathrm{H}), 5.84(1 \mathrm{H}, \mathrm{s}, \mathrm{Ar}-\mathrm{H}), 6.19(1 \mathrm{H}, \mathrm{d}, J=$ $7.64 \mathrm{~Hz}, \mathrm{Ar}-\mathrm{H}), 6.46(1 \mathrm{H}, \mathrm{d}, J=7.60 \mathrm{~Hz}, \mathrm{Ar}-\mathrm{H}), 6.63(1 \mathrm{H}, \mathrm{d}, J=7.61 \mathrm{~Hz}, \mathrm{Ar}-\mathrm{H}), 6.88(1 \mathrm{H}, \mathrm{d}, J$ $=7.62 \mathrm{~Hz}, \mathrm{Ar}-\mathrm{H}), 7.50-7.80$ (4H, m, DCHNQ), 8.02-8.30 (4H, m, DCHNQ); ${ }^{13} \mathrm{C} \mathrm{NMR}\left(\mathrm{CDCl}_{3}\right)$; $\delta$ 29.12, 31.55, 35.00, $35.24\left(2 \mathrm{CH}_{2}-\mathrm{CH}_{2}\right), 117.90,122.00,125.10,125.31,125.60,126.47$, $126.82,126.90,127.25,128.74,129.64,130.82$, 131.43, 131.61, 132.05, 132.41, 132.76, 133.33, $133.85,134.13,134.66,135.44,136.16,137.55,141.23,141.83,144.71,146.24$ (Ar-C), 180.45, 180.80, 181.90, $182.34(\mathrm{CO}) ; \mathrm{MS}(70 \mathrm{eV}): \mathrm{m} / \mathrm{z}(\%)=622\left[\mathrm{M}^{+4}\right](28), 620\left[\mathrm{M}^{+2}\right](18), 618\left[\mathrm{M}^{+}\right]$ (100), 616 (13), 584 (20), 529 (6), 428 (6), 394 (12), 377 (24), 351 (15), 309 (12), 274 (100), 264 
(24), 217 (18), 191 (8), 149 (20), 119 (30), 105 (16); Anal. Calcd for $\mathrm{C}_{36} \mathrm{H}_{24} \mathrm{Cl}_{2} \mathrm{~N}_{2} \mathrm{O}_{4}$ : C, 69.68; H, 4.06; N, 4.51. Found: C, 69.50; H, 4.00; N, 4.40\%.

2- $\mathrm{N}$-[4'(13'-Amino[2.2]paracyclophanyl)]amino-3-cyano-1,4-naphthoquinone (11). Red crystals (acetonitrile) (mp $120{ }^{\circ} \mathrm{C}, 27 \mathrm{mg}, 48 \%$ ); IR (KBr): $\widetilde{v}_{\max }=3410-3337 \mathrm{~cm}^{-1}\left(\mathrm{NH}, \mathrm{NH}_{2}\right)$, 3099-3020 (Ar-CH), 2960-2890 (aliph.-CH), 2209 (CN), 1695 (CO); ${ }^{1} \mathrm{H}$ NMR ( $\left.\mathrm{CDCl}_{3}\right) ; \delta 2.90$ $3.35\left(8 \mathrm{H}, \mathrm{m}, 2 \mathrm{CH}_{2}-\mathrm{CH}_{2}\right), 3.70\left(2 \mathrm{H}, \mathrm{br}, \mathrm{s}, \mathrm{NH}_{2}\right), 5.82(1 \mathrm{H}, \mathrm{d}, J=1.64 \mathrm{~Hz}, \mathrm{Ar}-\mathrm{H}), 6.08(1 \mathrm{H}, \mathrm{dd}, J$ $=7.70,1.65 \mathrm{~Hz}, \mathrm{Ar}-\mathrm{H}), 6.33(1 \mathrm{H}, \mathrm{dd}, J=7.71,1.64 \mathrm{~Hz}, \mathrm{Ar}-\mathrm{H}), 6.39-6.43$ (3H, m, Ar-H), 7.988.12 (4H, m, DCNQ), $11.00(1 \mathrm{H}, \mathrm{br}, \mathrm{s}, \mathrm{NH}) ;{ }^{13} \mathrm{C} \mathrm{NMR}\left(\mathrm{CDCl}_{3}\right) ; \delta 29.47,31.41,35.02,35.07$ (2 $\left.\mathrm{CH}_{2}-\mathrm{CH}_{2}\right), 112.78(\mathrm{CN}), 122.80,124.18,125.70,125.77,126.80,127.06,129.84,132.83$, $132.92,133.00,134.70,134.95,135.25,135.76,135.76,136.07,136.72,136.83,136.85,141.39$ (Ar-C), $179.16(\mathrm{CO}), 180.26(\mathrm{CO}) ; \mathrm{MS}(70 \mathrm{eV}): \mathrm{m} / \mathrm{z}(\%)=419\left[\mathrm{M}^{+}\right]$(20), 392 (34), 299 (14), 273 (100), 144 (14), 130 (20), 119 (78), 92 (10); Anal. Calcd for $\mathrm{C}_{27} \mathrm{H}_{21} \mathrm{~N}_{3} \mathrm{O}_{2}$ : C, 77.31; H, 5.05; N, 10.02. Found: C, 77.50; H, 4.95; N, 10.12\%.

\section{4-Amino-13- $\mathbf{N}, \boldsymbol{N}$-bis-2' -(3'-cyano-1,4-naphthoquinonyl)amino[2.2]paracyclophane}

(12). Orange crystals (ethanol) (mp $180{ }^{\circ} \mathrm{C}, 9 \mathrm{mg}, 22 \%$ ); IR (KBr): $\widetilde{v}_{\max }=3335 \mathrm{~cm}^{-1}\left(\mathrm{NH}_{2}\right), 3035-$ 2999 (Ar-CH), 2865-2820 (aliph. CH), 2226-2219 (CN), 1692 (CO); ${ }^{1} \mathrm{H}$ NMR $\left(\mathrm{CDCl}_{3}\right) ; \delta 2.90-$ $3.35\left(8 \mathrm{H}, \mathrm{m}, 2 \mathrm{CH}_{2}-\mathrm{CH}_{2}\right), 3.70\left(2 \mathrm{H}, \mathrm{br}, \mathrm{s}, \mathrm{NH}_{2}\right), 5.82(1 \mathrm{H}, \mathrm{d}, J=1.65 \mathrm{~Hz}, \mathrm{Ar}-\mathrm{H}), 6.08(1 \mathrm{H}, \mathrm{dd}, J$ $=7.68,1.70 \mathrm{~Hz}, \mathrm{Ar}-\mathrm{H}), 6.33(1 \mathrm{H}, \mathrm{dd}, J=7.67,1.65 \mathrm{~Hz}, \mathrm{Ar}-\mathrm{H}), 6.46-6.40$ (3H, m, Ar-H), 7.727.55 (4H, m, DCNQ), 8.15-6.97 (4H, m, DCNQ); ${ }^{13} \mathrm{C} \mathrm{NMR}\left(\mathrm{CDCl}_{3}\right) ; \delta 29.50,31.60,34.90$, $35.03\left(2 \mathrm{CH}_{2}-\mathrm{CH}_{2}\right), 114.50,116.00(2 \mathrm{CN}), 122.13,127.11,127.30,129.99,131.35,132.37$, $132.47,132.75,134.13,134.18,134.28,134.31,134.45,134.74,134.99,135.60,136.02,138.15$, 139.50, 141.12, 141.92, 145.10 (Ar-C), 175.50, 175.90, 180.20, 181.80 (4CO); MS (70 eV): m/z $(\%)=600\left[\mathrm{M}^{+}\right](60), 418(40), 392(20), 299$ (16), 238 (30), 119 (100), 104 (26); Anal. Calcd for $\mathrm{C}_{38} \mathrm{H}_{24} \mathrm{~N}_{4} \mathrm{O}_{4}$ : C, 75.99; H, 4.03; N, 9.33. Found: C, 75.85; H, 4.00; N, 9.50\%.

2-N-[4'(13'-Amino[2.2]paracyclophanyl)] amino-3-cyano-5,6-dichloro-1,4-naphthoquinone (13). Red crystals (benzene) (mp $\left.200{ }^{\circ} \mathrm{C}, 17 \mathrm{mg}, 32 \%\right)$; IR (KBr): $\widetilde{v}_{\max }=3410-3337 \mathrm{~cm}^{-1}(\mathrm{NH}$, $\mathrm{NH}_{2}$ ), 3015-3000 (Ar-CH), 2852 (aliph.-CH), 2209 (CN), 1690 (CO); ${ }^{1} \mathrm{H} \mathrm{NMR}\left(\mathrm{CDCl}_{3}\right) ; \delta 2.80-$ $3.10\left(6 \mathrm{H}, \mathrm{m}, \mathrm{CH}_{2}-\mathrm{CH}_{2}\right), 3.20-3.35\left(2 \mathrm{H}, \mathrm{m}, \mathrm{CH}_{2}-\mathrm{CH}_{2}\right), 3.50\left(2 \mathrm{H}, \mathrm{br}, \mathrm{s}, \mathrm{NH}_{2}\right), 5.80(1 \mathrm{H}, \mathrm{d}, J=$ $7.90 \mathrm{~Hz}, \mathrm{Ar}-\mathrm{H}), 6.15(1 \mathrm{H}, \mathrm{dd}, J=7.88,1.71 \mathrm{~Hz}, \mathrm{Ar}-\mathrm{H}), 6.40(1 \mathrm{H}, \mathrm{dd}, J=7.89,1.70 \mathrm{Hy}, \mathrm{Ar}-\mathrm{H})$, 6.58-6.45 (3H, m, Ar-H), $11.00(1 \mathrm{H}, \mathrm{br}, \mathrm{s}, \mathrm{NH}) ;{ }^{13} \mathrm{C} \mathrm{NMR}\left(\mathrm{CDCl}_{3}\right) ; \delta 29.70,31.40,33.60,35.71$ $\left(\mathrm{CH}_{2}-\mathrm{CH}_{2}\right), 112.78(\mathrm{CN}), 122.45,125.70,125.78,126.85,127.05,132.83,132.93,134.70$, $134.95,135.26,135.76,136.84,140.20,141.40,145.00,149.70$ (Ar-C), 179.30, 181.5 (2CO); $\operatorname{MS}(70 \mathrm{eV}): \mathrm{m} / \mathrm{z}(\%)=442\left[\mathrm{M}^{+4}\right](18), 440\left[\mathrm{M}^{+2}\right](20), 438\left[\mathrm{M}^{+}\right](100), 436(24), 404(38), 402$ (60), 400 (36), 300 (18), 274 (26), 272 (30), 230 (50), 132 (18), 119 (2), 104 (40); Anal. Calcd for $\mathrm{C}_{23} \mathrm{H}_{17} \mathrm{Cl}_{2} \mathrm{~N}_{3} \mathrm{O}_{2}$ : C, 63.03; H, 3.84; N, 9.59. Found: C, 62.90; H, 3.84; N, 9.50\%.

4-Amino-13- $\mathrm{N}, \mathrm{N}$-bis-2 ${ }^{\prime}$-(3'-chloro-5'-cyano-1,4-benzoquinonyl)amino-[2.2]paracyclophane (14). Brown crystals (ethanol) $\left(\mathrm{mp}>300{ }^{\circ} \mathrm{C}, 8 \mathrm{mg}, 20 \%\right)$; IR $(\mathrm{KBr}): \widetilde{v}_{\max }=3340 \mathrm{~cm}^{-1}\left(\mathrm{NH}_{2}\right)$, 3020-3000 (Ar-CH), 2850 (aliph.-CH), 2211 (CN), 1690 (CO); ${ }^{1} \mathrm{H}$ NMR $\left(\mathrm{CDCl}_{3}\right) ; \delta$ 3.30-2.82 $\left(8 \mathrm{H}, \mathrm{m}, 2 \mathrm{CH}_{2}-\mathrm{CH}_{2}\right), 3.82\left(2 \mathrm{H}, \mathrm{br}, \mathrm{s}, \mathrm{NH}_{2}\right), 5.86(1 \mathrm{H}, \mathrm{d}, J=7.85 \mathrm{~Hz}, \mathrm{Ar}-\mathrm{H}), 6.20(1 \mathrm{H}, \mathrm{dd}, J=$ 7.89, $1.76 \mathrm{~Hz}, \mathrm{Ar}-\mathrm{H}), 6.45(1 \mathrm{H}, \mathrm{dd}, J=7.91,1.75 \mathrm{~Hz}, \mathrm{Ar}-\mathrm{H}), 6.58-6.45(3 \mathrm{H}, \mathrm{m}, \mathrm{Ar}-\mathrm{H}) ;{ }^{13} \mathrm{C}$ 
NMR $\left(\mathrm{CDCl}_{3}\right) ; \delta 30.72,32.34,33.80,35.50\left(\mathrm{CH}_{2}-\mathrm{CH}_{2}\right), 117.26,115.80(2 \mathrm{CN}), 124.35,126.82$, $126.90,127.0,127.20,129.80,130.90,131.87,132.80,133.0,133.30,133.76,134.00,135.18$, 135.33, 137.20, 137.87, 141.40, 145.00, 149.70 (Ar-C), 179.50, 179.80, 188.40, 188.56 (4CO); MS (70 eV): m/z (\%) = $646\left[\mathrm{M}^{+8}\right](2), 644\left[\mathrm{M}^{+6}\right](6), 642\left[\mathrm{M}^{+4}\right](18), 640\left[\mathrm{M}^{+2}\right](38), 638\left[\mathrm{M}^{+}\right]$ (100), 636 (20), 612 (20), 510 (14), 508 (20), 486 (30), 390 (26), 276 (22), 132 (18), 104 (16); Anal. Calcd for $\mathrm{C}_{30} \mathrm{H}_{16} \mathrm{Cl}_{4} \mathrm{~N}_{4} \mathrm{O}_{4}$ : C, 56.45; H, 2.53; N, 8.78. Found: C, 56.55; H, 2.48; N, 8.65\%.

\section{References}

1. Singer, L. A.; Cram, D. J. J. Am. Chem. Soc. 1963, 85, 1080.

2. Cram, D. J.;. Bauer, R H. J. Am. Chem. Soc. 1959, 81, 5971.

3. Sheehan, M.; Cram, D. J. J. Am. Chem. Soc. 1969, 91, 3553.

4. Aly, A. A.; El-Tamany, S. H.; Mourad, A. E. Heterocyclic Commun. 1997, 3, 175.

5. Aly, A. A.; Hassan, A. A.; Mohamed, N. K.; Mourad, A. E. Pharmazie 1997, 52, 282.

6. Aly, A. A.; Mohamed, N. K.; Hassan, A. A.; Mourad, A. E. Bull. Chem. Soc. Jpn. 1996, 69, 2249.

7. Mohamed, N. K.; Hassan, A. A.;. Aly, A. A Mourad, A. E.; Hopf, H. J. prakt. Chem. 1996, $338,745$.

8. Hassan, A. A.; Mohamed, N. K.; Ibrahim, Y. R.; Sadek, K. Y.; Mourad, A. E. Bull. Chem. Soc.Jpn. 1993, 66, 2612.

9. Hassan, A. A.; Mohamed, N. K.; Ibrahim, Y. R.; Mourad, A. E. Liebigs Ann. Chem. 1993, 695.

10. Hassan, A. A.; Mohamed, N. K.; Ali, B. A.; Mourad, A. E. Tetrahedron 1994, 50, 9997, 10010.

11. Hassan, A. A.; Aly, A. A.; Mohamed, N. K. . Mourad, A. E J. Chem. Res. (S) 1996, 208.

12. Hassan, A. A.; Mohamed, N. K.; Aly, A. A.;. Mourad, A. E Monatsh. Chem. 1996, $128,61$.

13. Hassan, A. A.; Ibrahim, Y. R. . Semida, A. A Mourad, A. E. Liebigs Ann. Chem. 1994, 989.

14. Aly, A. A.; Hassan, A. A.; Mourad, A. E. Can. J. Chem. 1993, 71, 1845.

15. Aly, A. A.; El-Shaieb, K. M Tetrahedron 2004, 60, 3797.

16. Zitt, H.; Dix, I.; Hopf, H.; Jones, P. G. Eur. J. Org.Chem. 2002, 2298.

17. Chatterjee, S J. Chem. Soc. B 1969, 6, 725.

18. Bundi, M. L.; Jayadevappa, E. S. Spectrochim. Acta 1988, 44A, 607. 